\section{Relación entre la experiencia de caries dental e higiene bucal en escolares de la Provincia de Sechura-Piura en el año 2010.}

Caballero-García $\mathrm{C}^{1}$, Enriquez $\mathrm{G}^{2}$, García-Rupaya $\mathrm{C}^{3}$. Relación entre la experiencia de caries dental e higiene bucal en escolares de la Provincia de Sechura-Piura en el año 2010. Rev Estomatol Herediana. 2012; 22(1):16-19.

\section{RESUMEN}

Objetivo: Determinar la relación entre la experiencia de caries dental e higiene bucal en escolares de Sechura. Material y Métodos: El diseño del estudio fue de tipo transversal. La muestra fue aleatoria y estuvo constituida por 438 niños de edades entre 6 a 14 años, habitantes de la localidad de Sechura. El diagnóstico de salud oral fue realizado en noviembre de 2010 por los alumnos de una Escuela de Estomatología en Piura. Para la medición de la caries dental se utilizó el índice CPOD y ceod, para el diagnóstico de la higiene bucal se utilizó el IHO. Las pruebas de Chi-cuadrado y Odds ratio fueron utilizadas para el análisis estadístico de los datos. Resultados: Se encontró que los escolares de mayor edad tienen 3 veces más probabilidades de desarrollar caries dental $(\mathrm{OR}=3,253)$. Se determinó que existe relación entre la presencia de caries dental y el género de los niños $(\mathrm{p}=0,028)$ y también se encontró una relación estadísticamente significativa entre la presencia de caries dental y la higiene bucal $(p<0,001)$. Conclusiones: Esta investigación presenta el estado de salud bucal de localidades poco estudiadas en el Perú, lo cual servirá para establecer un plan de trabajo odontológico y un monitoreo adecuado para disminuir esta enfermedad.

Palabras clave: CARIES DENTAL, SALUD BUCAL, SALUD ESCOLAR, COMUNIDAD RURAL.

Relationship between the caries and deficient oral hygiene in school children from Sechura, Piura.

SUMMARY

Objectives: Determinate the relationship between the experience of dental caries and oral hygiene in Sechura's schoolchildren. Material and Methods: The study design was cross-sectional. A random sample of 438 children aged 6 to 14 was studied in Sechura, Piura. The screening of oral health diagnosis performed in November 2010 by the students of School of Dentistry in Piura university. For measure the dental decay was used the DMFT index and for the oral hygiene was used the IHO index. Chi-square test and odds ratio was performed for the data analysis. Results: The oldest schoolchildren are 3 times more likely to develop dental caries $(\mathrm{OR}=3.25)$. It was determined a relationship between the presence of dental caries and gender of children $(p=0.028)$ and also was found a significant difference of relationship between the presence of dental caries and oral hygiene $(\mathrm{p}<0.001)$. Conclusions: This investigation was corroborated the conceptions we already know and helps us to learn the oral health condition of different communities in Perú that have less opportunities of oral care, which will serve to establish an odontologic work plan and a right control to decrease dental caries.

Key words: DENTAL CARIES, ORAL HEALTH, SCHOOL HEALTH.

\section{Carmen Caballero García ${ }^{1}$ Guillermo Enriquez ${ }^{2}$ Carmen García Rupaya ${ }^{3}$}

${ }^{1}$ Bachiller en Estomatología. Directora de la Escuela de Estomatología - Universidad César Vallejo. Piura, Perú.

²Bachiller en Odontología. Docente de la Escuela de Estomatología - Universidad César Vallejo. Piura, Perú.

${ }^{3}$ Magíster en Estomatología. Universidad Peruana Cayetano Heredia. Lima, Perú.

\section{Correspondencia}

Carmen Stefany Caballero García Calle Laredo Mz. C Lote 2 San Miguel, Lima, Peru.

Teléfono: 511-981453017

E-mail:stefa135@gmail.com

\section{Introduccion}

Las enfermedades bucales son las más comunes de las enfermedades crónicas y son un importante problema de Salud Pública por su alta prevalencia, impacto en los individuos, en la sociedad y el costo de su tratamiento (1).

La aparición de la caries dental y la enfermedad periodontal, de no ser controladas pueden causar un desequilibrio en los tejidos bucales, propiciando alteraciones funcionales (2).

La OMS afirma que la caries dental, la enfermedad periodontal y el cáncer oral constituyen los mayores problemas globales que afectan la salud bucal en países industrializados, países en desarrollo $y$ en especial en las comunidades más pobres (3).

Powell en 1998, realizó una revisión sobre los modelos de predicción multifactorial de la caries dental; encontró que los predictores mas fuertes eran aquellos asociados con la edad y los indicadores de experiencia pasada de caries, lo cual fue indicado también por Mattos en el $2004(4,5)$.

En la literatura se ha descrito que el control de placa bacteriana es ambiguo en relación a la presen- cia y el desarrollo de caries cuando se han comparado solo ambas variables. La evidencia nos indica que la buena higiene bucal reduce la experiencia de caries $(5,6)$. Los pacientes que infrecuentemente $o$ ineficientemente limpian sus dientes y/o tienen pobre control manual pueden estar en alto riesgo de presentar esta enfermedad. La habilidad de realizar un buen cepillado puede también cambiar con el tiempo por diversas circunstancias. Es el odontólogo el que está en una posición ideal para detectar este cambio (7).

Los indicadores de riesgo de caries dental están asociados a la 
ocurrencia de la enfermedad y se relacionan a los estudios transversales $(4,5)$. Su identificación permite tomar acciones de control y prevención (8), estas actividades son realizadas por diversas facultades de Estomatología del norte del país, con la finalidad de establecer programas preventivos odontológicos que ayuden a la erradicación de estas enfermedades tan importantes y prevalentes de la cavidad oral en pacientes niños.

El presente estudio brinda datos que determinan el perfil epidemiológico de salud oral de esta población del Perú que ha sido poco estudiada; lo cual servirá para establecer programas preventivos promocionales y realizar un seguimiento epidemiológico de la población.

El propósito de esta investigación fue determinar la relación entre la experiencia de caries dental y la higiene bucal en escolares de la provincia de Sechura, Piura en el año 2010.

\section{Material y Métodos}

Se realizó un estudio de tipo transversal y descriptivo. La muestra estuvo constituida por 438 escolares que correspondieron a niños de las localidades de La Bocana, Parachique, Ciudad del Pescador y Puerto Rico todas pertenecientes a la provincia de Sechura en el departamento de Piura.

Los padres de los niños consintieron que sus hijos participen en la investigación constituyendo así la totalidad de la muestra. De igual manera los niños asintieron su participación en el estudio.

Los estudiantes cumplieron con los siguientes criterios de selec- ción: Ser residentes de estas localidades, ser escolares de entre 5 a 12 años, y asistir de manera voluntaria a la valoración de su diagnóstico de salud realizado en el mes de Noviembre de 2010 por la Escuela de Estomatología de la Universidad César Vallejo.

El método para determinar las condiciones de salud bucal fue la observación directa. Se empleó lo índices de CPOD y ceod para de- terminar la experiencia presente y pasada de caries dental; y el índice IHO propuesto por Löe y Silness para determinar la presencia de placa dentobacteriana y cálculo dental. Para la exploración bucal se emplearon espejos planos del número 5 y exploradores estandarizados de una sola punta, se empleo luz natural. La exploración se hizo siguiendo los criterios establecidos por la Organización Panamericana de la Salud (OPS) (15).
Grafico 1. Presencia de caries dental en escolares de la Provincia de Sechura - Piura en el año 2010.

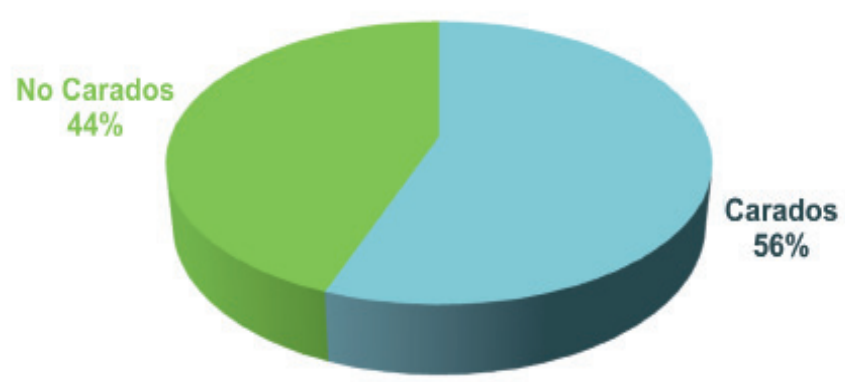

Gráfico 2. Indiec de higienc oral en escolares de la provincia de Sechura-Piura en el año 2010 .

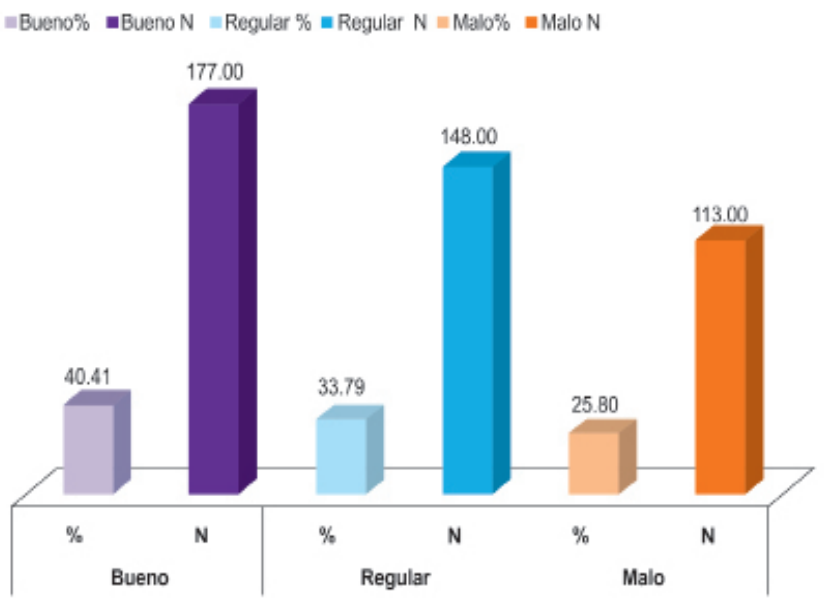


Los datos fueron analizados en el programa Microsoft Excel y posteriormente se realizaron los cálculos estadísticos mediante el paquete SPSS 15.0. Para el análisis descriptivo de la investigación se obtuvo medidas de frecuencia y para hallar la relación de las variables de estudio se realizó la prueba de Chi cuadrado y la determinación del Odds ratio.

\section{Resultados}

Se encontró que un $56 \%$ de escolares presentaron caries dental en la población de estudio (Figura 1).

El índice de higiene oral mostró que el $40 \%$ de los escolares presentan una buena higiene bucal mientras que el $26 \%$ presenta el nivel más bajo (malo) de este índice (Figura 2). Los niños de mayor edad (9-12 años) tienen más probabilidad de presentar caries que aquellos escolares menores según esta investigación (Tabla 1).

Se encontró una relación significativamente estadística $(p=0,028)$ entre la presencia de caries dental y el género de los escolares, en donde los estudiantes de género masculino tuvieron mayor proporción de presencia de caries dental que los de género femenino (Tabla 2).

No se encontró asociación entre el índice de higiene oral y las variables edad y género (Tablas 3 y 4).

Finalmente, se encontró una relación estadísticamente significativa $(p<0,001)$ entre la presencia de caries dental y la higiene bucal de los niños (Tabla 5). Los escolares con índice de higiene oral regular y mala presentaron mayor proporción de presencia de caries dental que los escolares con Índice de Higiene oral buena.

\section{Discusión}

El presente estudio se realizó en la provincia de Sechura en el depar-

tamento de Piura con una muestra conformada por 438 niños en etapa escolar.

En el Perú la población menor de 14 años constituye el $41 \%$ del

Tabla 1. Asociación entre la presencia de caries dental y grupo etáreo.

\begin{tabular}{lll|l}
\hline & & & \\
& & & \multicolumn{2}{c}{ Intervalo de confianza al } & $\mathbf{9 5 \%}$ \\
\cline { 3 - 4 } & & Inferior & Superior \\
\cline { 3 - 4 } $\begin{array}{l}\text { Razón de las } \\
\text { ventajas para } \\
\text { grupo etáreo }(5- \\
8 / 9-12)\end{array}$ & 3,253 & 2,194 & 4,822 \\
\hline
\end{tabular}

Tabla 2. Relación entre la presencia de caries dental y género.

\begin{tabular}{lll}
\hline Género & \multicolumn{1}{c}{$\begin{array}{c}\mathbf{N}^{\mathbf{0}} \\
\text { cariados }\end{array}$} & Cariados \\
\hline Masculino & 86 & 134 \\
Femenino & 108 & 110 \\
Total & 194 & 244
\end{tabular}

Prueba de chi cuadrado $\mathrm{p}=0,028$
Tabla 5. Relación entre la presencia de caries dental y el índice de higiene oral.

\begin{tabular}{ccc}
\hline IHO & No cariados & Cariados \\
\hline Bueno & 100 & 77 \\
Regular & 52 & 96 \\
Malo & 42 & 71 \\
Total & 194 & 244 \\
\hline
\end{tabular}

Prueba de chi cuadrado $\mathrm{p}=0,000$
Tabla 3. Relación entre índice de higiene oral y grupo etáreo.

\begin{tabular}{cccc}
\hline \multirow{2}{*}{ Grupo etáreo } & \multicolumn{3}{c}{ IHO } \\
\cline { 2 - 4 } & Bueno & Regular & Malo \\
\hline $5-8$ años & 85 & 60 & 54 \\
9 - 12 años & 92 & 88 & 59 \\
Total & 177 & 148 & 113 \\
\hline
\end{tabular}

Prueba de chi cuadrado

$\mathrm{p}=0,340$

Tabla 4. Relación entre índice de higiene oral y género.

\begin{tabular}{lccc}
\hline \multirow{2}{*}{ Grupo etáreo } & \multicolumn{3}{c}{ IHO } \\
\cline { 2 - 4 } & Bueno & Regular & Malo \\
\hline Masculino & 79 & 74 & 64 \\
Femenino & 98 & 74 & 46 \\
$\quad$ Total & 177 & 148 & 113 \\
\hline $\begin{array}{l}\text { Prueba de chi cuadra- } \\
\text { do }\end{array}$ \\
$\mathrm{p}=0,05$
\end{tabular}


total (INEI, 1981), considerándose ésta una población importante a ser estudiada por sus problemas de salud, los niños en la etapa escolar comprendida entre los 6 y 12 años de edad se encuentran en proceso de recambio dentario y constituyen también las edades en que las necesidades de atención odontológica van en ascenso (9).

Al igual que Moreno y cols (10), en el presente estudio se encontró que existe una relación significativa $(p<0,001)$ entre la presencia de caries dental y la edad de los estudiantes. De igual forma se encontró una fuerte asociación entre estas dos variables $(\mathrm{OR}=3,253)$.

Según diferentes estudios, la caries afecta más a la población masculina $(11,12)$, corroborando también los resultados de esta investigación $(p=0,028)$. Raitio $y$ cols. mencionaron que los indicadores de riesgo para caries dental no son iguales para los niños que para las niñas (13). Los resultados sugieren que existe una necesidad de identificar los factores que están impactando en los perfiles de caries encontrados en la población de estudio.

Franco no encontró relación entre la higiene oral y la edad reafirmándose así el resultado en esta investigación (14). Sin embargo, si halló diferencias significativas al relacionar las variables higiene oral $\mathrm{y}$ género, al igual que el resultado obtenido en este estudio $(p=0,05)$.

En cuanto a los factores asociados a la caries, este estudio corrobora la importancia de la higiene bucal, ya que la relación entre ambas variables fue estadísticamente significativa $(p<0.001)$. La higie- ne oral se ha identificado como un factor de riesgo desde hace varias décadas $(16,17,18)$ por lo que es necesario insistir en los programas de educación y prevención en salud oral.

Este tipo de investigación reafirma los conceptos ya conocidos en la evidencia científica publicada, y presenta el estado de salud bucal de los niños de esta localidad del norte del país, orientando sus resultados a elaborar un plan estratégico de trabajo odontológico y un monitoreo adecuado para disminuir esta enfermedad.

\section{Referencias Bibliográficas}

1. Sheiham A. Oral health, general health and quality of life. Bull World Health. 2005; 83 (9): 644.

2. Greene JC, Suomi JD. Epidemiology and public health aspects of caries and periodontal disease. $\mathrm{J}$ Dent Res. 1977; 56:20-6.

3. World Health Organization. Who releases new report on global problem of oral diseases. Geneva: World Health Organization; 2004.

4. Mattos M, Melgar M. Riesgo de caries dental. Rev Estomatol Herediana. 2004; 14(12):101-6.

5. Powell V. Caries prediction: a review of the literature. Community Dent Oral Epidemiol. 1998; 26: 361-71.

6. Demers M, Brodeur J, Simard P, Mouton C, Veilleux G, Fréchette S. Caries predictors suitable for mass-screenings in children: a literature review. Community Dent Health. 1990; 7:11-21.

7. Kidd E. Assessment of caries risk. Dent Update. 1998; 25: 385-90.

8. Daly B, Watt R, Batchelor P, Treasure E. Essential dental public health. New York: Oxford University Press; 2003.

9. Instituto Nacional de Estadística e Informática. Censos Nacionales
1981 y 1993. Lima: INEI; 2008.

10.Pineda M, Castro R, Watanabe V, Chein V, Ventocilla H. Necesidades de tratamiento para caries dental en escolares de zonas urbano y urbano marginal de Lima. Odontol sanmarquina. 2000; 1(6): 26-32.

11.Moreno A, Carreón G, Alvear G, López M, Vega F. Riesgo de caries en escolares de escuelas oficiales de la ciudad de México. Rev Mex Pediatr. 2011; 68(6): 228-33.

12.Milen A, Hausen H, Heinonen O, Paunio I. Caries in primary dentition related to age, sex, social status, and county of residence in Finland. Dent Oral Epidemiol. 1981; 9: 83-6.

13.Kerosuo H, Honkala E. Caries experience in the primary dentition of Tanzanian and Finnish 3-7 year old children. Community Dent Oral Epidemiol. 1991; 19: 272-6.

14.Raitio M, Pienihakkinen K, Scheinin A. Multifactorial modeling for prediction of caries increment in adolescents. Acta Odontol Scand. 1996; 54: 118-21.

15. Franco C. Prevalencia de caries $\mathrm{y}$ gingivitis en preescolares. CES Odontol. 1995; 8(2): 128-31.

16.Leverest D, Proskin H, Feathersone J, et al. Caries risk assessment in a longitudinal discrimination study. J Dent Res. 1993; 72(2): 538-43.

17.Clark B, Graves R, Webster D, Triol C. Caries and treatment patterns in children related to social class in children related to school lunch program eligibility. Journal of Public Health Dent 1987; 43(3): 134-38

18.Petersen PE. Sociobehavioural risk factors in dental caries - international perspectives. CommunHity Dent Oral Epidemiol. 2005; 33(4):274-9. 